\title{
Short Versus Longer Term Effectiveness of an Outreach Program for the Homeless Mentally III ${ }^{1}$
}

\author{
Deborah Bybee \\ Michigan State University \\ Carol T. Mowbray \\ Wayne State University School of Social Work
}

\section{Evan Cohen}

The University of Michigan Medical Center Department of Physical Medicine and Rehabilitation

Presents 12-month follow-up results from an outreach/linkage intervention with persons who are homeless and mentally ill, contrasting these with results obtained at 4 months. Both sets reflect the success of the program in placing individuals in independent housing. However, longer term data provide useful information regarding client movement patterns and increased tenure in nonhomeless living arrangements beyond the termination of specialized services. Analyses of 12-month residential outcomes identified four variables as significant predictors: recruitment source, project service duration, CMH service duration, and client age. In contrast to 4-month predictors, variables reflecting baseline client functioning were no longer significantly related to outcome, suggesting that the positive effects of the intervention may take longer to achieve with some clients. Discussion focuses on the implications of these effectiveness results for future research designs and measures as well as the utility and limitations of preexperimental approaches for evaluating innovative service models when implementation and efficacy experiences are lacking.

KEY WORDS: outreach; homeless; mentally ill.

${ }^{1}$ This research was supported in part by a grant from the National Institute of Mental Health, \#H87 MH44373, to the Michigan Department of Mental Health. 
Public attention to the national problem of homelessness and mental illness has increased in recent years - one outcome being an increase in intervention studies. As a result of the knowledge gained, some consensus has been reached about effective services for those who are homeless and mentally ill (Federal Task Force, 1992; Newman, 1992). It seems accepted that serving this population requires assertive outreach efforts; engagement is often challenging. We also know that providing housing alone is not sufficient. Supportive assistance to maintain housing is a necessity - persons with severe mental illness who have experienced homelessness are likely to again become unhoused unless there is continuity of needed services, because of the unpredictability and variability of their illness. However, due to the heterogeneity and diversity of this population and the multiplicity of their problems, a range of interventions and a variety of service providers are required, tailored to individual needs.

Although there is consensus on general principles of service for individuals who are homeless and mentally ill, agreement on effective intervention models is still lacking. Inadequate and inconsistent research methodologies have contributed to this information gap, as have difficulties in conceptualizing expected outcomes (Newman, 1992). Bybee, Mowbray, and Cohen (1994) and Newman (1992) both suggest that even the best interventions for persons who are homeless and mentally ill may have limited effects on distal outcomes reflecting the course of a mental illness (e.g., hospitalization, symptoms, etc.). A number of studies have demonstrated impacts on residential and service utilization variables, but not on functioning level or psychiatric measures (Blankertz \& Cnaan, 1992; Bybee et al., 1994; Lipton, Nutt, \& Sabatini, 1988). Mercier, Fournier, and Peladeau (1992) indicated that the primary outcome for research on persons with severe mental illness and/or substance abuse problems should be the type and pattern of domicile over time.

Another significant measurement and design issue concerns the length of time required for an intervention to demonstrate a significant impact. It is becoming more apparent, for the overall population of persons with severe mental illness, that improved outcomes are not quickly achieved. For example, the Nine City Robert Wood Johnson initiative to improve services for the chronically mentally ill failed to find client impacts at 12 -month followup, although significant system changes had been established (Goldman, 1992). Unfortunately, evaluation research studies on services to persons who are homeless and mentally ill have oftentimes employed short-term follow-up periods. Most have used 6 or 9 months (Barrow, Hellman, Lovell, Plapinger, \& Streuening, 1989; Caton, Wyatt, Grunberg, \& Felix, 1990; Goering, Wasylenki, St. Onge, Paduchak, \& Lancee, 1991; Rife, First, Greenlee, Miller, \& Feichter, 1991; Toomey, First, Rife, \& Belcher, 1989). But a number of 
projects have reported on much shorter follow-up intervals of 3 months or less (First, Rife, \& Kraus, 1990; Leda \& Rosenheck, 1992; Rosenheck \& Gallup, 1991). Few reports in the literature have utilized follow-up periods of 12 months or more (Lipton et al., 1988) and even then many have only reported on participant status (e.g., whether they were still in housing or services), without direct assessment of the participants themselves (Marcos, Cohen, Nardacci, \& Brittain, 1990; Toomey et al., 1989). It may be that researchers feel they need to sacrifice longer term follow-up periods to achieve higher follow-up rates. However, multiple follow-up time points can achieve both aims. Surprisingly, only a study reported by Toomey et al. (1989) collected follow-up data at more than one time point; unfortunately, only the 6-month results were reported.

The present study contrasts the short and longer term outcomes observed in an outreach and intervention project targeted to individuals who were homeless and mentally ill. It encompassed two sites and multiple recruitment sources to produce results with greater potential for generalizability than other studies. It also achieved high rates of follow-up success in tracking and locating service recipients ( $86 \%$ at 4 months; $83 \%$ at 12 months), thus decreasing the likelihood that artifacts of client attribution could be mistaken for program effectiveness (E. H. Cohen et al., 1993). The conceptual framework for the study's research design specified that the major outcome variable of interest was the extent and duration of clients' living in permanent-type, independent settings in the community; and consequently minimizing the utilization of institutional settings (hospitals, shelters and jails) (Mowbray et al., 1992). It was recognized that, for some clients, improvements in community functioning were necessary in order to increase the likelihood of maintaining a residence. However, it was acknowledged that major functioning level changes required long-term, sustained intervention - for the most part, beyond the scope of this outreach/linkage effort. Finally, the project expected that, given the challenging nature of the target population, there should be some relationship between positive client outcomes and amount of services provided (although it was not clear whether this should be a linear or some form of nonlinear relationship).

The current article presents analyses involving residential status as well as community functioning measures for a 12-month follow-up period; these findings are contrasted with 4-month results. Analyses also examined the relationship between mental health service utilization variables and outcomes. We then discuss implications of the findings vis-à-vis the success of this model as well as the necessary duration of interventions with persons who are homeless and severely mentally ill. We also discuss ramifications of the results for future research designs studying interventions with this target population. 


\section{METHODS}

\section{Description of the Intervention}

The Mental Health Linkage intervention model (Mowbray et al., 1992) was the basis for this NIMH-funded research demonstration. Sited in two Michigan communities, Factorytown and Collegetown, it utilized a team (4 to 5 full-time equivalent staff) of mental health workers to outreach to persons who were mentally ill and homeless or potentially homeless. The goal of the project was to house persons in independent residences of their choice in the community (rather than dependent care placements) as soon as possible, to provide them with support and assistance necessary to maintain a residence, and (once living arrangements and any extreme behavior problems were stabilized) to then transition them to ongoing community service systems (mental health, social services, etc.). Eligible clients were offered a variety of services, in vivo, by outreach workers: a comprehensive assessment of functionality, housing preferences, and needs; assistance in obtaining temporary and/or permanent housing; help in establishing income supports, including payee services; training or rehabilitation in activities of daily living and interpersonal/social skills which might affect independent living; mental health clinical services; and short-term intensive case management. Clients could receive any or all of these services, based on their own preferences, project staffs' assessment of needs, and the extent of their engagement in services. To optimize the likely appropriateness and effectiveness of services, clients were encouraged to participate in joint service planning. When housing was stabilized and immediate service needs met, with their consent, clients were referred from the project to mainstream community mental health $(\mathrm{CMH})$ (nonproject) therapists and/or case managers. Homeless Project staff assisted clients in this transition, to try and make the referral "stick," and thus discontinue clients' homeless project involvement. However, clients could be re-referred to the project or themselves request further services at any time. Staff resources were also utilized in locating and accessing independent housing sites and working with landlords to maintain housing opportunities.

\section{Eligibility and Recruitment}

Each site recruited participants from three types of settings: shelters, hospitals serving public mental health inpatients, and the existing $\mathrm{CMH}$ caseloads of aftercare clients. Participants recruited from any of the settings were screened by project staff (based on contact with the person and/or 
information from records, agency staff, etc.) and considered eligible if they presented serious mental illness, extreme residential instability, and county residency, and if their next living arrangements had not already been determined. Serious mental illness was defined to include psychotic disorders (thought or major affective disorders) or severe personality disorders which had entailed multiple admissions to inpatient or community acute care settings within the last year. The engagement process varied substantially across individuals and included describing the project to clients, meeting with them, offering services and assistance, and beginning the process of assessing needs. The project achieved a relatively high success rate in engaging clients screened eligible for services, in that $73 \%$ overall accepted some form of project assistance (Mowbray, Cohen, \& Bybee, 1993).

\section{Research Design}

The evaluation design examined residential status (where participants were living) at the two follow-up time points, as well as residential experiences and utilization of shelter and mental health services over the 12 months before and after homeless project enrollment. It also compared functioning level characteristics of individuals served at baseline with those same characteristics at 4- and 12-month follow-up. Finally it sought to determine to what extent characteristics of the person or of the intervention related to residential status. Feasibility and costs were considerations in the selection of this longitudinal panel design. We also felt, given our limited knowledge about effective treatment approaches for this population, that the research should serve as a preexperimental search for intervention features and client and setting characteristics related to outcome differences, to better identify elements that could be productively included in future experimental designs. Since experimental designs are expensive in terms of time and resources, expending funds for such research on noneffective interventions may be detrimental to long-range research and service goals.

\section{Sample}

All individuals accepting some help between October 1, 1989 and December 31, 1990 were included in this study. Of these 163 participants, $60.7 \%$ were from Factorytown and $39.3 \%$ from Collegetown. About equal numbers were recruited from hospital psychiatric inpatient units (36.2\%) and the CMH caseload (38.7\%), with somewhat fewer recruited from shelters $(25.2 \%)$ (Although it should be noted that individuals found at the 
shelter who were on the $\mathrm{CMH}$ caseload were assigned to the latter recruitment source.) The study participants were relatively young ( $M$ age $=37.5, S D=11.0)$, predominantly male $(57.1 \%)$ and white $(58.9 \%)$, with a substantial number (28.8\%) identified at screening as having substance-abuse problems.

\section{Data Collection}

Data were gathered through four methods: referral forms, client interviews, ratings by outreach workers, and examination of archival records. Basic demographic information and a detailed chronology of residential history for the prior 12 months was provided at the time of screening and referral through use of a standardized screening instrument. Intake interview data were collected by project outreach workers, who were trained by the project director and the second author. Signed informed consent and release of information forms were obtained for all participants included in the research.

Outreach workers completed a shortened version of the Client Level Assessment Measure (CLAM; Hazel, Herman, \& Mowbray, 1991) to assess client intake functioning levels. This instrument was derived from a longer interview (used in a statewide study of about 2,500 seriously mentally ill clients) through selection of items found to be most predictive of major scale scores along with those most relevant for planning housing arrangements. The CLAM variables included Community Living Needs, Depression, Deviancy, Psychoticism, Aggression, Financial Management Needs, and Psychotropic Medication Management Needs. All scales had internal consistencies of $\alpha=.64$ or above. (See Mowbray, Bybee, \& Cohen, 1993, for a more extensive description of client characteristics and measures.)

For the 4- and 12-month postintake follow-up data collection, trained research assistants (graduate students in the social sciences) conducted interviews with participants and obtained functioning assessments from raters most familiar with each client: homeless project outreach workers or CMH case managers and occasionally relatives or other care providers. Residential history data was also collected by research assistants from $\mathrm{CMH}$ and homeless project records and from interviews with project staff, family members, and clients themselves. Data on shelter and psychiatric hospital utilization during the 12-month baseline and 12month follow-up periods were obtained archivally, through reviews of the records of facilities utilized by public mental health clients in the two counties. Information was also accessed from the computerized 
management information systems of the two $\mathrm{CMH}$ Boards to produce two types of measures characterizing utilization of both homeless project services and mainstream $\mathrm{CMH}$ services: (a) duration of service provision (in months) over the 12-month baseline and follow-up periods and (b) intensity of service contacts, measured in hours per month while involved in services.

From the data collected, residences at each time period were characterized as homeless/correctional settings (shelters, street, jail or prison, temporary room), treatment-type settings (formal mental health or substance abuse inpatient, acute care, or residential programs), or permanenttype settings. The latter included independent living (either alone, with relatives or with nonrelated others) and supervised dependent settings (such as group homes or adult foster care).

\section{Attrition Rates and Characteristics}

For 4- and 12-month follow-ups, respectively, $23(14 \%)$ and $28(17 \%)$ participants could not be located (additionally, 2 were deceased at 4months and a total of 3 at 12 months). Successfully locating participants at both time points was significantly and positively related to full versus limited engagement in services and negatively to having lived alone or outside the local county for any period during the baseline year. Additionally, a positive screening of substance-abuse problems at intake was significantly related to nonlocatability at 4 months only. No other variables were related to follow-up locatability (age, gender, race, diagnosis, use of homeless shelters, hospitalizations, etc.).

\section{Data Analysis}

The framework guiding the analysis plan was to first examine available descriptive data on residential patterns postintervention to examine whether or not the project's overall goals had been met; for example, the extent to which clients utilized project services, were quickly housed in permanent settings, moved from these settings, and/or relocated to settings considered less desirable. Second, we examined changes over time in the primary and secondary dependent variables of interest (e.g., residential status and functioning levels). Third, we utilized multivariate techniques to explore relationships of residential outcomes with clients' baseline characteristics/histories as well as with intervention descriptors. 
Short-term (4-month) results from this project have been previously reported in detail (Bybee et al., 1994). Briefly, at 4-month follow-up, 57\% of service recipients were in permanent-type independent settings, $23 \%$ were in supervised dependent settings, $9 \%$ were in treatment facilities, and $11 \%$ were homeless or in corrections facilities. Three significant multivariate predictors of follow-up residential status were found: (a) recruitment source - those from shelters were more likely to be in independent settings alone or in temporary treatment or homeless settings, while those from hospitals were more likely to be in independent settings alone and those from $\mathrm{CMH}$ caseloads were more likely to be in supervised dependent care; (b) baseline functioning - those whose ratings assigned them to the "hostile psychotic" cluster were more likely than those in the "best functioning" cluster to be in supervised dependent or temporary treatment or homeless settings and less likely to be in independent settings alone; and (c) amount of homeless project servicethose receiving more hours of service were more likely to be in independent settings alone and less likely to be in temporary treatment or homeless settings. Additionally, while project site, race and substance-abuse problem showed marginally significant relationships, no aspect of baseline residential history or mental health service use predicted residential status at 4-month follow-up.

\section{RESULTS}

Results are first presented descriptive of residential experiences and service use at 4 versus 12 months, then of changes in residential status, service use, and functioning. Finally, multivariate analyses are presented to predict 12-month residential status.

\section{Description of Residential Experiences and Service Use}

Data were analyzed on overall patterns of participants' residential experiences and service utilization; that is, residential settings occupied at the 4- versus 12-month follow-up periods; cumulative residential experiences over the follow-up period; and service utilization.

\section{Residential Setting at 4- and 12-Month Follow-up}

For 132 of the 163 individuals in the follow-up cohort, it was possible to determine the types of situations in which they were living at 12-month follow-up. These are listed in Table I, compared with residential status at 4 months (from Bybee et al., 1994). 


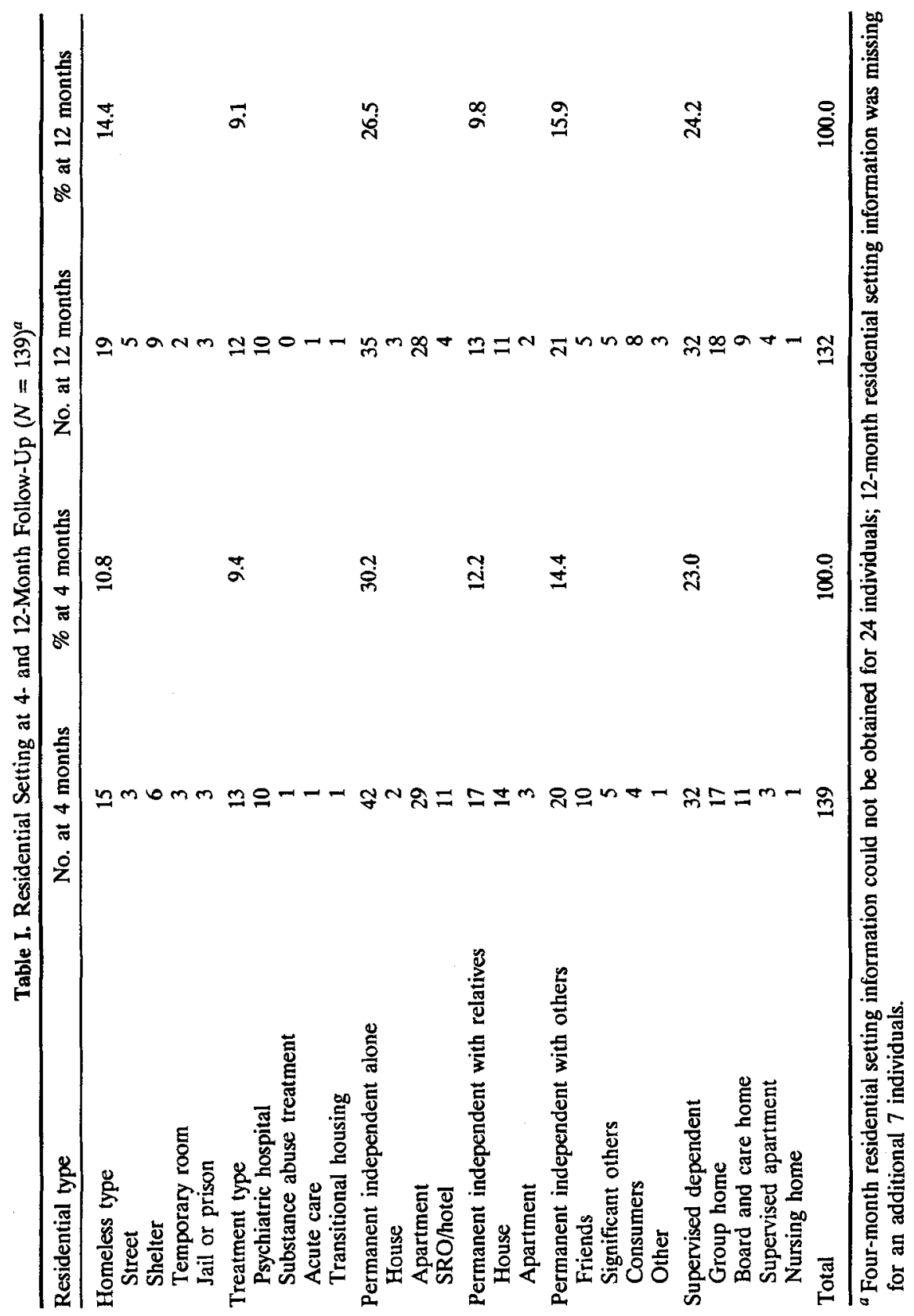


At 12 months, more than half of the participants were in permanent independent settings (most living alone, some with relatives or with others); one quarter in supervised dependent settings; and the remainder in treatment facilities or in homeless or correctional settings. Making the extreme assumption that all individuals who could not be located at each follow-up point were homeless, it was known that at least $76 \%$ of the entire group were not homeless at 4 months, and $71 \%$ were not homeless at 12 months. Cursory inspection of the marginal distributions in Table I shows remarkable similarity from 4- to 12 -month follow-up.

However, as Table II shows, the similarities in marginal distributions obscure substantial individual movement across the two time points. Across most types of settings (with treatment settings an exception), individuals were most likely to be in the same type of residence at 12 months that they were in at 4 months (Cohen's $\kappa=.40, p<.05$ ). However, nearly half of those who could be located at 12 months were living in a different type of arrangement than they were at 4-month follow-up. Although log-linear modeling found no global model of residential movement that fit the data, some meaningful trends could be discerned. Individuals who were in a homeless-type setting at 4-month follow-up were about equally likely to be homeless or in some type of independent setting at 12-month follow-up; none of them were in treatment at 12 months. Those in treatment settings at 4-month follow-up were most likely to have moved to supervised dependent care at 12 months, although about a quarter were still in treatment settings. Nearly three fourths of those in an independent setting at 4 months were in an independent setting at 12 months, although there were some changes in living partners. Very little change was seen among individuals in supervised dependent care at 4 months; they were least likely to be in homeless settings at 12 months.

\section{Cumulative Residential Experiences}

For $80 \%$ of the follow-up cohort, it was possible to ascertain where they had lived during 9 or more of the 12 postintake months. (For 123 individuals, housing information was complete for the entire 12-month period; between 1 and 3 months of missing information was imputed for 8 others.) During the entire 12-month follow-up, $82 \%$ of the cohort had some experience with permanent independent housing; the average person spent more than 6 months of the follow-up year in such settings, although variability among individuals was high. Forty-five percent spent some time in supervised dependent settings, with an average stay of 2.5 months. Sixtynine individuals $(53 \%)$ spent some time in a homeless or correctional setting 


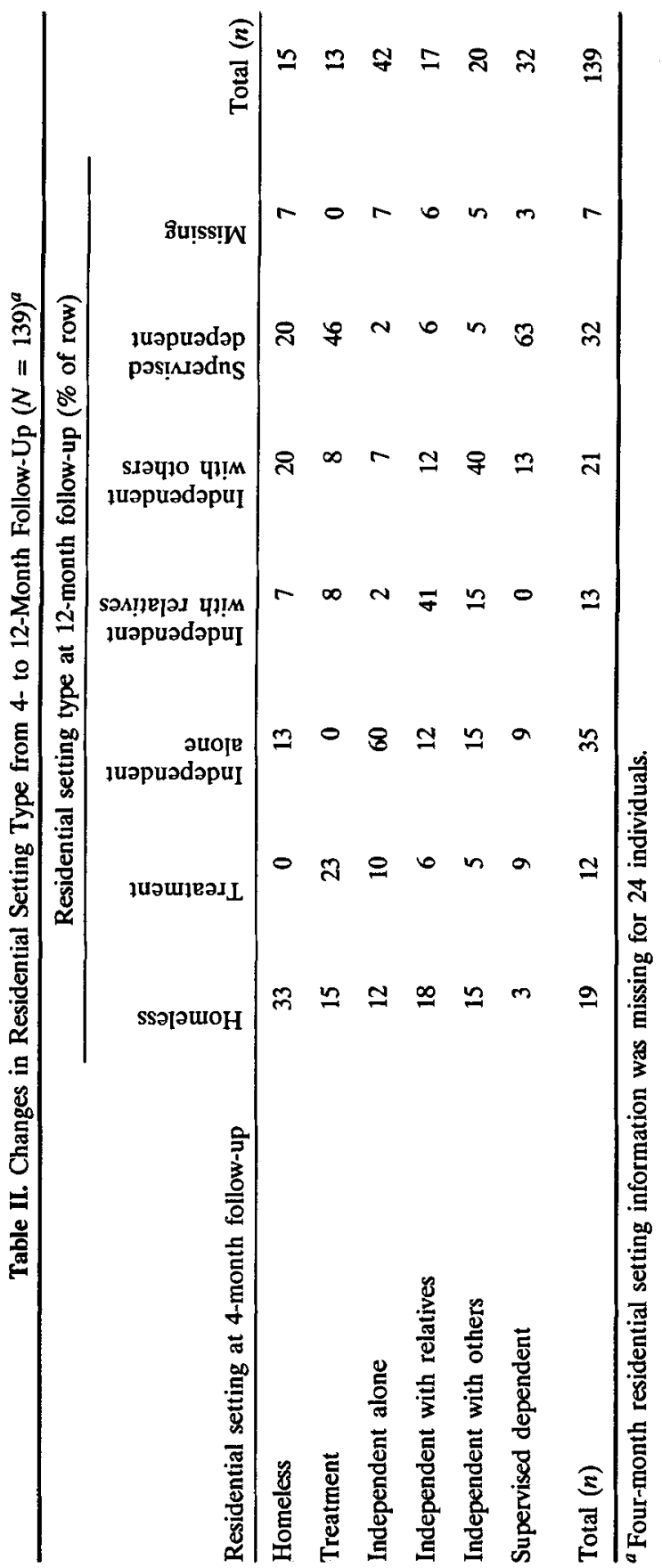


during the entire 12-month follow-up period, with an average total stay of 40 days. However, for nearly one third, these stays occurred before they obtained their first permanent-type residence. Of the individuals who obtained permanent housing, $51(36 \%)$ were known to have had subsequent episodes of homelessness or incarceration. All but 3 of these had street, shelter, or temporary room stays after their first permanent-type setting; 10 had jail or prison stays. Eight-four individuals (64\%) spend part of the 12-month follow-up in treatment settings, with an average total stay of 33 days. Of the 142 individuals who obtained permanent-type housing, 66 (46\%) subsequently had days in treatment settings (46 in psychiatric hospitals or hospital units, 19 others in acute care residential settings; 2 in inpatient substance abuse facilities).

Most individuals were placed in permanent-type housing relatively quickly: $78 \%$ (of 142) were placed within 30 days of first accepting project help in the community; for only $7 \%$ did the process take more than 4 months. It was also possible to examine the first housing placements for participants. Sixty-nine percent (of $N=163$ ) were initially placed in independent settings (52\% alone, $20 \%$ with relatives, and $28 \%$ with others), and $31 \%$ were placed in supervised dependent care. For the 31 individuals missing residential information at 4 or 12 months, it was known that 18 spent no time in permanent-type settings before they disappeared.

Everyone moved at least once during the follow-up year. Eight (6\%) moved only once, in all cases into a permanent-type setting in which they stayed for the remainder of the follow-up period; 22 individuals $(17 \%)$ moved more than 12 times. The greatest turnover in residences occurred during the first $\mathbf{3 0}$ days after placement in a permanent-type setting, during which $35 \%$ left their first housing. Only $31 \%$ remained in their first permanent-type housing more than 4 months or were still there at the conclusion of the 12-month follow-up. Although moving from one residence to another was common, moving outside the geographic area was not: $85 \%$ had no noninstitutional stays outside the local county.

\section{Service Utilization}

Homeless project intervention lasted an average of 2.5 months, with an average intensity of 4.75 hours of face-to-face or phone contact per enrolled month. Variability was high on both dimensions, with duration ranging from less than 1 up to 12 months and intensity ranging from less than 1 to nearly 25 hours per enrolled month. 


\section{Changes in Residential Status, Service Use, and Functioning}

Table III presents data separately on residential and service use experiences for follow-up months 1 through 4 versus 5 through 12 , as well as statistical comparisons between the two periods. The doubly multivariate repeated measures ANOVA is significant. During the latter follow-up period, individuals spent more days per month in permanent settings (both independent and supervised) and fewer days per month in treatment settings, although the number of individuals with experience in these settings was not significantly different for any type except supervised dependent care. While the decline in days per month in homeless or corrections settings was not statistically significant, the proportion of individuals with any days in such settings did decrease significantly. Individuals moved less often (and fewer moved at all) during months 5 through 12, but they became more geographically mobile, spending, on average, more days per month outside the county. As expected, homeless project contact decreased substantially after 4-month follow-up, in both duration (proportion of months) and intensity (hours per month); however, contrary to expectation, involvement in mainstream $\mathrm{CMH}$ services did not increase significantly following termination of homeless project involvement. Continuing involvement with either the homeless project or mainstream $\mathrm{CMH}$ services showed no relationship with residential change from the 4-month to the 12-month interval; no time by service involvement interactions were found.

Staff ratings of functioning at 12-month follow-up were available for 126 individuals in the follow-up cohort. To check for change in functioning over time, doubly multivariate repeated measures analysis of variance was performed on assessments at project intake, 4-month follow-up, and 12month follow-up. No significant change could be detected by this method. Because idiosyncratic missing data caused a serous reduction in $n$ (to 42 in analyses involving all scales over three time points), univariate tests, using Bonferonni adjusted probability levels, were also performed. No meaningful changes in functioning level measures were identified.

\section{Prediction of Residential Setting Type at 12-Month Follow-Up}

\section{Demographic and Intervention Variables Related to 12-Month Follow-Up Residential Setting}

Multinomial logistic regression (Agresti, 1990) was used to identify predictors of residential setting type at 12 -month follow-up. For this analysis, settings with relatives and with others were combined to yield four 


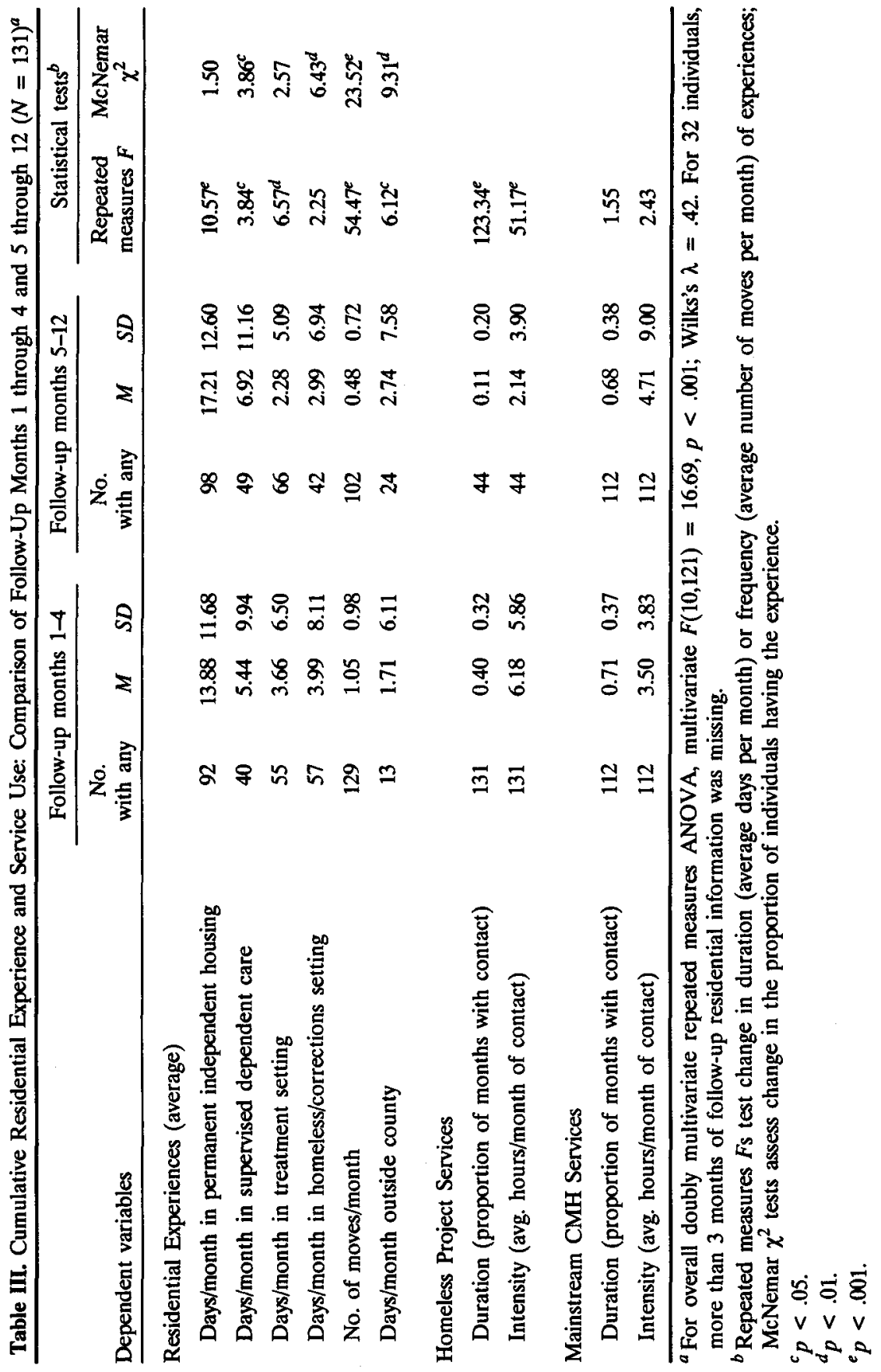


comparative groups of similar size: independent setting, alone; independent setting, with others; supervised dependent setting; and temporary setting (treatment or homeless). All comparisons were with the temporary setting group. Results may be found in Table IV.

Four variables were significant predictors of being in a permanent setting (compared with temporary) at 12 months: age group, recruitment source (entered as two dummy variables with $\mathrm{CMH}$ caseload recruitment at the comparative category), duration of involvement with Homeless Project services (in months), and duration of involvement with mainstream CMH services (during the postintake period of months 5-12. Overall, the model including these variables was significantly predictive of the data, although it accounted for a modest proportion of the variance (McFadden's $\rho^{2}=.130$ ). (For this $R^{2}$ analog, a transformation of the LR $\chi^{2}$, values between .2 and .4 are generally considered substantial; Hensher \& Johnson, 1981.)

The first section of Table IV lists the effect of each predictor on the odds ratio of each type of permanent setting compared with a temporary (homeless or treatment) setting. Specifically, holding other effects constant, age had a positive effect on the odds that an individual would be in either an independent alone or a supervised dependent setting at 12-month follow-up. Each increase in age group from young (30 and under), to middle (31 to 40), to older (over 40) nearly tripled the odds of being in an independent alone setting and multiplied by 2.5 the odds of being in supervised dependent care. Compared with individuals recruited from the $\mathrm{CMH}$ caseload, those from inpatient psychiatric hospitals had nearly 11 times the odds of being in an independent setting alone at 12-month follow-up rather than in a temporary setting.

Intensity of service contact (hours of contact per month), either with the Homeless Project or with mainstream $\mathrm{CMH}$ services, showed no significant impact on the odds of any of the permanent setting types compared with a temporary setting at 12 -month follow-up. However, duration (in months) of involvement with services, both those provided by the Homeless Project and those of the mainstream CMH agency, had a positive effect on the odds of being in a permanent setting at 12-month follow-up.

The individual variable derivatives section on Table IV shows the change in probability of each residential-type associated with a one unit change in each independent variable (Steinberg \& Colla, 1991). In contrast with the odds ratio in the first section of this table, which in each case reflects a comparison with the odds of being in a temporary-type residential setting, the derivatives reflect absolute changes in the probabilities of each type of residential setting. For example, recruitment from a homeless shelter 


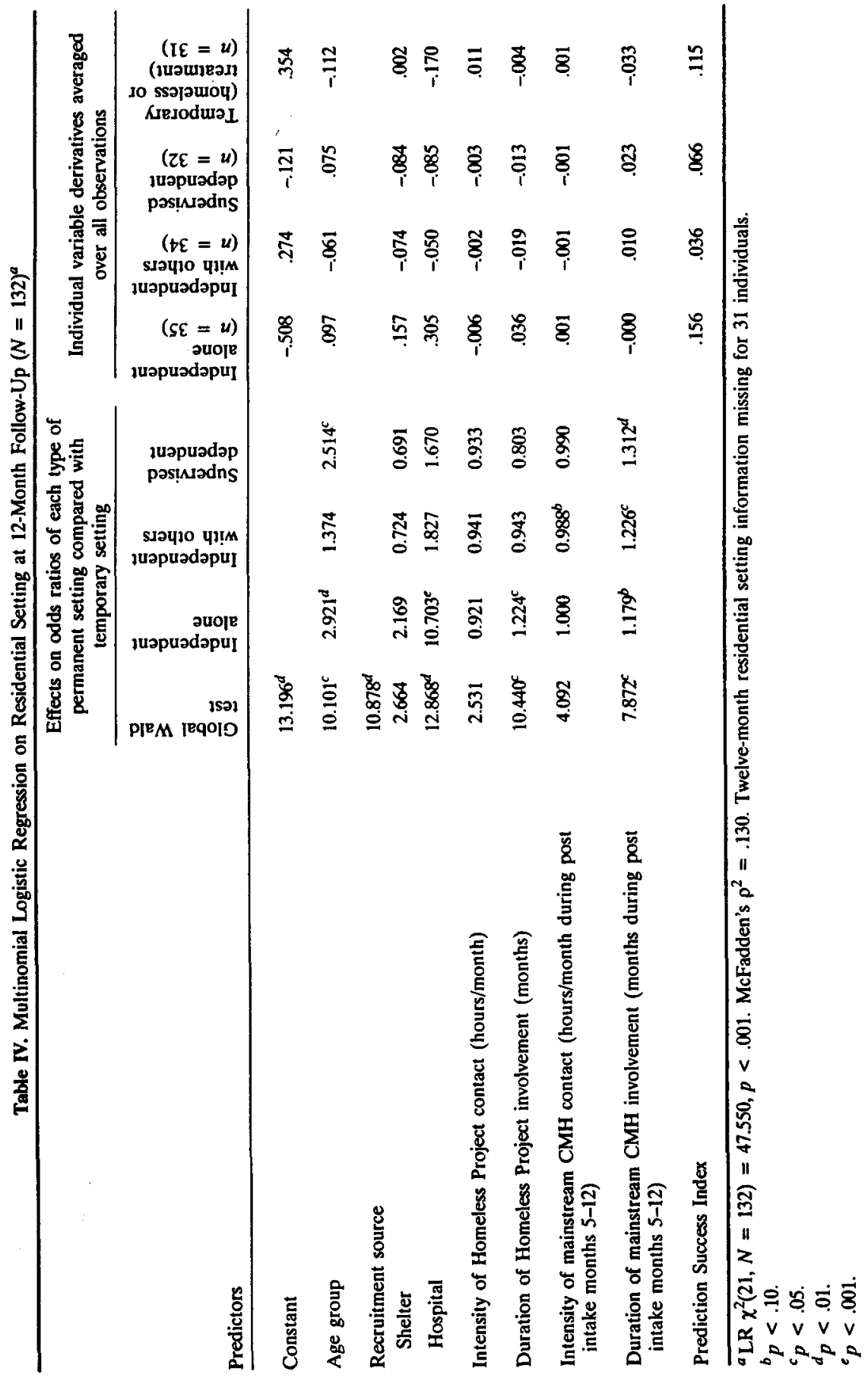


increased the probability of being in an independent alone setting (by .16) while decreasing the probability of an independent setting with others (by .07 ) and the probability of supervised dependent care (by .08). Duration of Homeless Project involvement increased the probability of an independent alone setting at 12-month follow-up (by .04 for each additional month of involvement) while decreasing the probability of an independent setting with others (by .02 for each month) and the probability of supervised dependent care (by .01 for each month). Additionally, duration of mainstream CMH service involvement decreased the probability of a temporary (homeless or treatment) setting (by .03 for each month of involvement) while increasing the probability of supervised dependent care (by .02 for each month) and the probability of an independent setting with others (by .01 for each month). For each residential setting type, Table IV lists a prediction success index, an indication of the degree to which the regression model improved on chance prediction. Improvements, although modest for all types, were somewhat stronger for the prediction of independent alone and temporary settings; they were especially slight for independent settings with others.

\section{Variables Not Contributing to Prediction of 12-Month Follow-Up Residential Setting}

Several variables that made significant or marginally significant contributions to the prediction of residential setting type at 4-month follow-up showed no significant relationship with type of residence at 12-month follow-up: project site, race, identification of a substance abuse problem at project intake, and baseline functioning cluster. As at 4-month follow-up, baseline residential history variables (days in treatment settings, supervised dependent settings, permanent independent settings, and homeless settings; number of moves; number of days outside the local county) and archival baseline measures of shelter use, psychiatric hospital use, and CMH services use showed no relationship with 12-month residential setting type. Additionally, no significant interaction effects among the significant predictors (age, recruitment source, intervention duration) were found.

\section{Covariates of Cumulative Residential Experience and CMH Service Utilization During Follow-Up Months 5 through 12 Postintake}

While the above analysis explored covariates of residential status at the point of the follow-up snapshot, it could not reveal much about 
cumulative housing and service experiences over the course of the follow-up period. Set correlation (J. Cohen, 1982, 1989) was used to explore relationships between baseline, demographic and intervention variables and cumulative outcomes such as amount of time in various types of residential settings and utilization of mainstream $\mathrm{CMH}$ services. This analytic approach allowed for an analysis of partial variance, examining relationships between a set of intervention descriptors and a set of outcome variables after statistically removing from each the potentially confounding effects of a set of baseline variables. Outcome experiences were aggregated over the postintake months 5 through 12 , a period following the termination of homeless project services for two thirds of participants. Assessing outcomes over this interval reduced the effects of intervention artifacts such as continued shelter stays while an individual was awaiting receipt of entitlement benefits or reduced use of mainstream $\mathrm{CMH}$ services while an individual was receiving homeless project intervention.

Initial analyses examined whole set associations between residential and service use outcomes and three sets of background variables. Two sets involving demographic and baseline functioning variables failed to show significant multivariate correlations with the cumulative outcome measures, (i.e., there were no overall outcome differences by sex, race, age, substance abuse problem, or baseline functioning level). However, the set of baseline residential and $\mathrm{CMH}$ service use variables (days in permanent independent, supervised dependent, treatment, and homeless or corrections settings plus hours of mainstream $\mathrm{CMH}$ service during the baseline year) was significantly related to the set of cumulative outcomes, Rao $F(42,505)=1.739, p<.01$, indicating that a significant portion of variation in follow-up residential and service use patterns could be explained by baseline history on these same indicators. Specifically, individuals with more days in permanent independent settings during their baseline year spent more days in independent residences during the follow-up period; those with more days in homeless or corrections settings during baseline spent fewer days in supervised dependent care during follow-up and had lower intensity $\mathrm{CMH}$ contact (fewer hours per month) during follow-up; those with more hours of $\mathrm{CMH}$ service during baseline were involved with $\mathrm{CMH}$ services for more months during the follow-up period. The magnitude of the overall relationship between sets was not particularly large, accounting for less than $10 \%$ of the (additive) variance in the outcome measures.

The set of variables descriptive of the Homeless Project intervention (project site, recruitment source, intervention intensity and duration) also showed a significant overall unpartialled relationship with the set of 
cumulative residential and service use outcomes, $\operatorname{Rao} F(63,654)=1.56$, $p<.005$. Although this analysis indicated that intervention differences were associated with differences in outcome, it could not rule out the possibility that the association might simply reflect relationships between intervention and baseline variables. Partialling was used to statistically remove these potentially confounding effects. Table $\mathrm{V}$ presents the results, that is, the effects of intervention on follow-up measures after the effects of baseline covariates have been removed from both sets. The overall partial association between intervention and follow-up was marginally significant, Rao $F(63,564)=1.300, p<.07$. However, given the small sample and resultant low power for this complex multivariate analysis (estimated at 0.64 for the detection of a multivariate $R^{2}{ }_{Y, X}$ of .20 at $\alpha=.05$ ), relaxation of the standards of statistical significance seems justified. Inspection of the results indicates that duration of Homeless Project involvement showed a strong positive relationship with follow-up days in permanent independent settings, a strong negative relationship with follow-up days in supervised dependent settings, and a marginally significant negative relationship with follow-up days in treatment settings. Graphical inspection of the significant interaction between site and duration of intervention isolated these effects to the Collegetown site; no outcome differences due to intervention duration were found at the Factorytown site.

The analysis also revealed a significant relationship between follow-up days in homeless or corrections settings and the interaction of shelter recruitment and Homeless Project contact intensity. Graphical examination of this interaction effect on the residuals revealed a negative association between contact intensity and follow-up days in homeless or corrections settings; no significant relationship was found for individuals recruited from other settings. Among individuals recruited from shelters, those receiving higher intensity interventions had fewer follow-up days in homeless or corrections settings than would have been predicted from their baseline days in such settings.

The effects of interaction terms such as the above should be interpreted with caution, given the small sample size, the relatively large number of variables involved, and the marginal significance of the overall analysis. Interpretation of the interaction effects, especially those involving recruitment source, were based on small cells (29 in the case of individuals recruited from shelters) and may be unstable on cross-validation. It is possible that three-way interactions involving project site, recruitment source, and intervention intensity or duration might contribute to understanding outcome differences. Unfortunately, with the available sample, it was not possible to test such complex effects. 


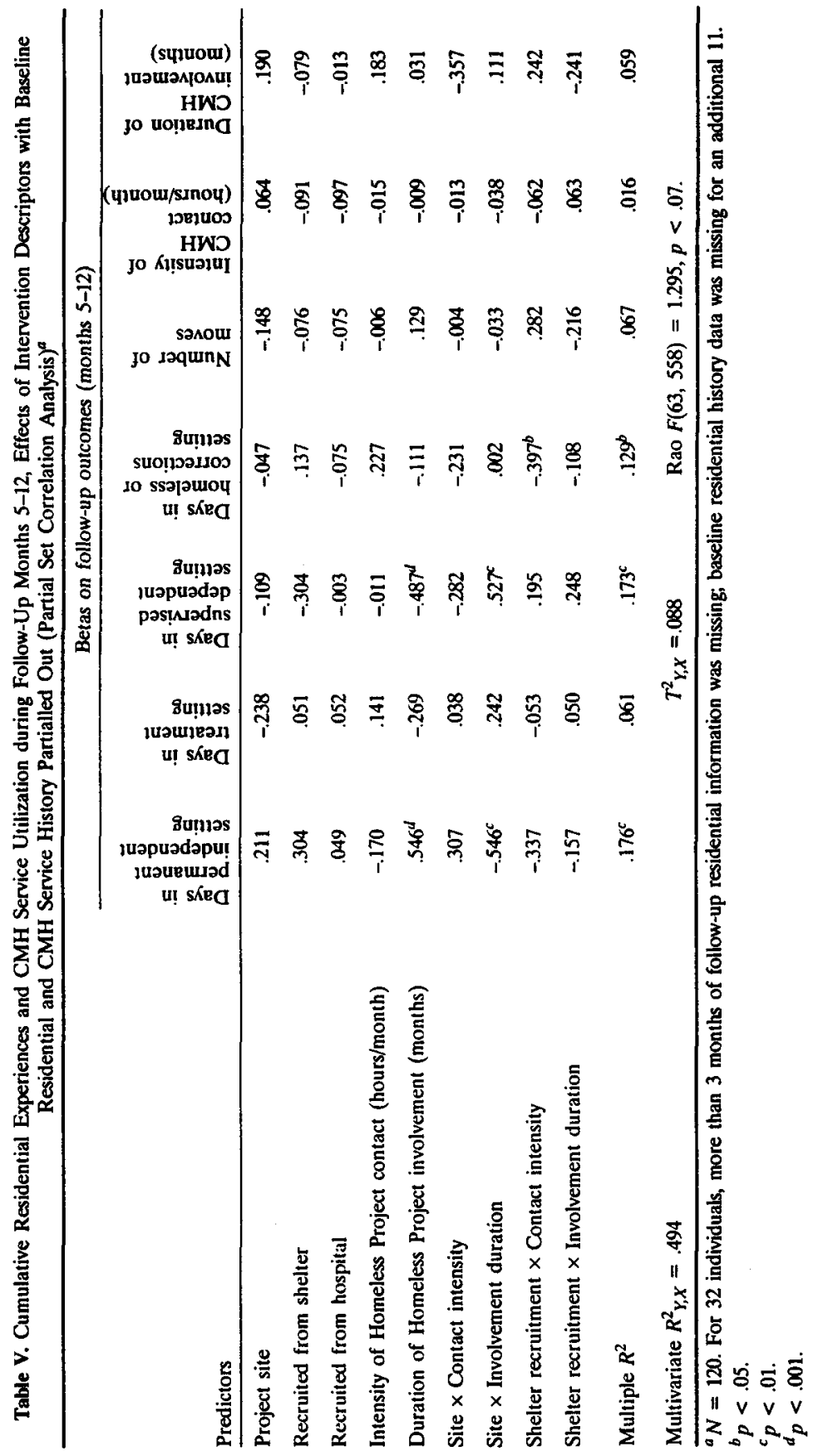




\section{DISCUSSION}

The results are discussed in terms of comparison with short-term (4 month) outcomes, implications for research on service interventions, and contextural and systems-level issues, with conclusions concerning ramifications for designing research on homelessness and mental illness.

\section{Short Versus Longer Term Effects}

The descriptive analyses of the status of participants at 4 versus 12 months paint similar pictures. That is, at both time periods, a majority of participants had exited from their homeless state, with 81 and $87 \%$, respectively, having spent some time in a permanent-type residence. Although the number of individuals who remained at their first permanent-type placement had declined over the additional 8 months (from 42 to 15\%), snapshot descriptions of participants' residences at 4 versus 12 months were quite similar, with few in homeless settings or treatment settings; the majority in independent settings; and about a quarter in supervised dependent care. At neither time point were there significant improvements over baseline on functioning level measures. Overall, this aggregate comparison might suggest that a 12-month follow-up produces no more enlightenment than a 4-month, other than to establish that gains made during project involvement were essentially maintained.

However, further examination showed substantial residential movement between the follow-up points. Of those who were homeless or in a treatment facility at 4-month follow-up, $60 \%$ were in permanent housing at 12-month follow-up; those in treatment at 4 months were most likely to be in supervised dependent housing at 12 and those homeless at 4 months most likely to be in an independent setting. Movement from these settings is contrasted with that from supervised dependent care and independent alone housing, in which the majority of individuals were stable.

Analysis of the cumulative residential experiences of participants also illuminates differences. There was a significant increase in the average days per month in permanent independent housing, supervised dependent care, and days outside the county as well as a decrease in the average days per month in treatment and in the number of moves per month. There was also a significant decline in the number of individuals who had any days in homeless or corrections settings. Overall, these changes indicate continued residential improvement extending beyond the termination of homeless services. 
The analyses predicting residential setting type at 4 months versus 12 months also showed some differences. At the shorter follow-up, three variables accounted for a substantial proportion of the variance in residences: recruitment source, hours of service from the homeless project, and functioning cluster type. Individuals who received more project services and those recruited from shelters or inpatient units were more likely to be living independently alone, while those characterized as "hostile-psychotic" were less likely to be in those settings. The latter group was more likely to be living in supervised dependent care or in temporary settings. Those recruited from hospitals were less likely to be in supervised dependent care. Finally, those recruited from shelters were also more likely to be in temporary settings.

At 12 months, the model significantly predictive of residential outcome included four variables as significant predictors. Recruitment source was a factor but was more limited in its effects to those from the hospital setting. The other variables were: duration of homeless project service contact, duration of contact with ongoing CMH services, and age. No client functioning data were related to residential status at 12 months.

In a more stringent test of unconfounded association, duration of project intervention was related positively to follow-up days in permanent independent settings and negatively to days in supervised dependent settings (but only for one site). With variation in baseline residential and service use history statistically removed, the set of variables describing homeless project intervention showed a marginally significant overall relationship with cumulative residential outcomes over 12 months. For participants recruited from shelters, higher intensity interventions were associated with fewer follow-up days in homeless or corrections settings.

Thus, 12-month data provided additional clues as to the "success" mechanisms of the Mental Health Linkage model. First, baseline clientfunctioning measures (hostile/psychotic cluster descriptors) were no longer significant predictors of residential outcome. This may imply that some clients difficult to work with at first may still achieve positive residential outcomes within 12 months. The same intervention seems to work with these clients as with other types; however, it takes longer to demonstrate its effects. Reliance on 4-month data might have suggested that a different approach was needed.

Second, clients recruited from shelters were among those most likely to have days living in temporary settings (homeless, correctional) at 4 and 12 months. However, analyses at the latter time point suggests that at least when taking these individuals' residential histories into account, 
more intensive project contacts were related to more successful residential outcomes. This effect may have shown up at 12 months because more cumulative residential data and/or project service data were available for analysis, or because positive effects took longer to be evidenced. Again, the implications are that over 12 months the intervention worked for these difficult individuals as well, compared to 4 months data suggesting a lack of effectiveness.

Thus, based on our experiences, investment in follow-up data collection extending to 12 months and including two time points seems appropriate for future intervention studies with persons who are homeless and mentally ill. It should be noted, however, that even at 12 months, no significant changes were found on functioning-level measures. This may reflect the insensitivity to change of the instruments we utilized, or it may suggest that even longer follow-up periods (e.g., 18 or 24 months) may better tap changes in domains more distal to the intervention.

\section{Implications for Future Research on Service Intervention}

Within the limitations of the design, data collected suggest promise for the effectiveness of the Mental Health Linkage model. That is, during the course of the project, a higher percentage of clients served $(87 \%)$ were placed in independent-type settings than had been reported in other interventions with this population: $67 \%$ for a New York City study (Barrow, Hellman, Lovell, Plapinger, \& Struening, 1991), 57\% in Ohio (Toomey et al., 1989); and 80\% in Minneapolis (Sosin, Pilliavin, \& Westerfelt, 1990). Although success criteria for homeless projects have not been established, these results are certainly promising. Multivariate analysis contribute to this conclusion, in that service variables were significantly related to permanenttype housing outcomes.

Of course, appropriate interpretation of the results from this preexperimental research requires attention to several caveats. First, without a control group, one cannot be certain that the apparently high rates of permanent housing at follow-up are actually higher than one would expect without intervention. Second, without randomized control over elements of the intervention, one cannot be certain of the causal direction of associations with outcome. For instance, it cannot be proven whether an intervention of longer duration "caused" a greater likelihood of living in an independent alone setting, whether being in an independent alone setting "caused" a longer intervention, or whether individuals who obtained independent alone housing were 
more likely to accept or request longer interventions. Third, with the sample available to this study, power for detecting differences between groups was relatively low and the potential for overfitting complex multivariate models to the idiosyncracies of the sample was relatively high. However, the results at least indicate the desirability of further replications of Mental Health Linkage, with improved research designs. Suggestions for these research improvements can also be gleaned from the project's results.

Besides the service variables, the data analyses at 12 months identified several other significant predictors of residential status. Recruitment source was a major predictor. As speculated in the 4-month analysis (Bybee et al., 1994), this variable appears to be a proxy for other client characteristics. These do not appear to include baseline functioning, since such measures were not significantly related to housing outcome. Several possibilities might be suggested. First, recruitment source may reflect the individual's stable functioning rather than functioning measured at project intake, when individuals were in flux due to recent hospitalization or housing crisis. Features of the recruitment source might lead one to expect stable functioning to be (a) higher than intake functioning among those recruited from acute hospitalizations; (b) similar to intake functioning among those referred from the $\mathrm{CMH}$ caseload; and (c) most variable among shelter recruits. Indeed, some evidence for these patterns was found: From intake to 4-month follow-up, the most substantial trends toward improvement in functioning were noted for the hospital group, the least change for the $\mathrm{CMH}$ group, and the greatest variability for the shelter group. These trends paralleled follow-up residential patterns, with hospital recruits more likely to be in independent alone settings, $\mathrm{CMH}$ referrals in supervised dependent care, and shelter recruits in either independent alone or temporary settings. Future research could benefit from attempts to reliably measure individuals' usual functioning, not just their status when they enter a program. This might be done by contacting collaterals or by repeating functioning-level assessments over a short period.

Alternatively, recruitment source may reflect satisfaction with traditional helping programs and/or willingness to be involved in formal mental health service systems. That is, homeless persons who have severe mental illness and are found in shelters (versus those who enter a psychiatric hospital or who are currently on a $\mathrm{CMH}$ caseload) may be those who are avoiding the mental health system, due to prior negative experiences or because they feel mental health is not relevant to their problems. This possibility seems related to conclusions about the need to fully engage clients who are homeless and mentally ill in order to produce the most positive outcomes (Dennis, Buckner, Lipton, \& Levine, 1991; Drake, Osher, \& Wallach, 
1991). This interpretation implies that future research on homeless interventions should better conceptualize and measure extent of engagement, utilizing it to interpret differential outcomes or to assess differential levels of service intensity and contact required.

Age was also a significant predictor, with older age increasing the odds of living in permanent type settings at 12 months. This may reflect the fact that older adults have more stable life patterns, or that entitlement programs are more often available to them (Brickner \& Scanlan, 1990). Future research results might be more interpretable when combined with qualitative methods like ethnography or case histories to provide more detailed information about particular subgroups of interest.

Descriptive data on residential movements also have important implications. One example is the relative lack of movement of individuals in supervised dependent care settings. These living arrangements seem to reflect a person's age, as well as ratings of their community living skills. However, literature on supported housing approaches suggests they may also reflect the preferences of mental health workers to utilize structured settings that produce few demands on them, although they may not be most appropriate for the clients' needs and/or preferences (Carling, 1992). Indeed, the match between a client's preferred housing and the permanent housing they obtain appears, from some studies, to be an important determinant of successful outcomes (e.g., Barrow et al., 1989). This project did collect consumer preference data (see Yeich, Mowbray, Bybee, \& Cohen, 1994, for a description). However, it was not possible to also collect detailed data about the housing obtained in order to assess the extent of match with initial preferences. Future research on homeless interventions should develop measures in this area.

Finally, it should be noted that both 4- and 12-month analyses reflect a modest ability for the research data to predict housing status. Apparently, more variables affect an individual's living arrangement than we have directly or indirectly measured. Some suggestions as to the nature of these variables were provided through the analyses of residential movement. For example, there is a trend for more individuals to move out of independent settings living with relatives and living with others than is the case for the other permanent-type settings. Our research and our interventions may need to pay more attention to contextural variables: for example, the social support provided by and/or needed by those who are living with these homeless mentally ill clients. Regarding relative caretakers, the National Alliance for the Mentally Ill has asserted that deinstitutionalization in many instances has resulted in relatives being given too much responsibility for the care and housing of severely mentally ill family members (Hyde \& Goldman, 1993; Lefley, 1993). 


\section{Context and Systems Level Issues}

Despite its apparent success in placing individuals in permanent-type settings, the project still observed clients experiencing an overall high number of moves per month, with great variability in residential stability noted for some individuals. This suggests that issues larger than client-level interventions may need to be addressed. All those involved with attempting to better the outcomes of persons who are homeless and mentally ill need to be continually reminded of the economic and community limitations affecting our interventions and the barriers posed to the efforts of disabled individuals to improve themselves. That is, the availability and acceptability of community housing that is safe and affordable continues to be an issue for all poor people, and even more so for those identified as having a disability. In an overview of program evaluation challenges concerning services to the homeless mentally ill, Mercier et al. (1992) pointed out the need to understand that such services are embedded in a community context, suggesting a need to evaluate missing pieces, and noting that participation in the programs we are studying represents "no more than a single element in a complex process of social reintegration [for our clients]" (p. 422). Evaluators and service providers must recognize the limitations of their designs and efforts and act accordingly: both towards expanding the context of data collection, to include measures of support systems, the availability and receipt of desired housing, and the existence of other community limitations, and towards social action to remedy these larger system problems. As other researchers have noted, ". . . people simply cannot be healthy if they do not have a stable place to live ..." (Wright, 1990, p. 31).

\section{Conclusions}

For the Mental Health Linkage Project, the results of this service demonstration provide useful information for a future experimental study. First, strategies have been identified to improve the efficiency of implementation (see Mowbray, Cohen, \& Bybee, 1991). A more costly research demonstration of this model, utilizing random assignment to a control group, will be able to start up faster, with greater fidelity to the model, from its initiation. Second, we have identified needed improvements in measurement approaches. Third, the results give us some confidence that this model can be effective across heterogeneous subgroups, but that some of these groups, overall, can be expected to require interventions of longer duration or greater 
intensity. Our experience with this demonstration project shows both the benefit of multiple, follow-up time points and also the utility of a preexperimental design in refining intervention and research components and in identifying associational hypotheses for subsequent experimental test.

\section{REFERENCES}

Agresti, A. (1990). Categorical data analysis. New York: Wiley.

Barrow, S. M., Hellman, F., Lovell, A. M., Plapinger, J., \& Struening, E. L. (1989). Effectiveness of programs for the mentally ill homeless. New York: New York State Psychiatric Institute.

Barrow, S. M., Hellman, F., Lovell, A. M., Plapinger, J. D., \& Struening, E. L. (1991). Evaluating outreach services: Lessons from a study of five programs. In N. L. Cohen (Ed.), Psychiatric outreach to the mentally ill. New directions for mental health services (No. 52, pp. 29-46). San Francisco: Jossey-Bass.

Blankertz, L. E., \& Cnaan, R. A. (1992). Principles of care for dually diagnosed homeless persons: Findings from a demonstration project. Research on Social Work Practice, 2, 448-464.

Brickner, P. W., \& Scanlan, B. C. (1990). Health care for homeless persons: Creation and implementation of a program. In P. W. Brickner et al. (Eds.), Under the safety net (pp. 3-14). New York: Norton.

Bybee, D., Mowbray, C. T., \& Cohen, E. (1994). Evaluation of a homeless mentally ill outreach program: Differential short-term effects. Evaluation and Program Planning, in press.

Carling, P. J. (1992). Homes or group homes? Future approaches to housing, support and integration for people with psychiatric disabilities. Adult Residential Care Joumal, 6(2), 87-96.

Caton, C. L. M., Wyatt, R. J., Grunberg, J., \& Felix, A. (1990). An evaluation of a mental health program for homeless men. American Journal of Psychiatry, 147, 286-289.

Cohen, E. H., Mowbray, C. T., Bybee, D., Yeich, S., Ribisl, K., \& Freddolino, P. P. (1993). Tracking and follow-up methods for research on homelessness. Evaluation Review, 17, 333-354.

Cohen, J. (1982). Set correlation as a general multivariate data-analysis method. Multivariate Behavioral Research, 17, 301-341.

Cohen, J. (1989). SETCOR: A supplementary module for SYSTAT and SYGRAPH. Evanston, IL: SYSTAT, Inc.

Dennis, D., Buckner, J. C., Lipton, F. R., \& Levine, I. S. (1991). A decade of research and services for homeless mentally ill persons: Where do we stand? American Psychologist, 46, $1129-1138$.

Drake, R. E., Osher, F. C., \& Wallach, M. A. (1991). Homelessness and dual diagnosis. American Psychologist, 46, 1149-1158.

First, R. J., Rife, J. C., \& Kraus, S. (1990). Case management with people who are homeless and mentally ill: Preliminary findings from an NIMH demonstration project. Psychosocial Rehabilitation Journal, 14(2), 87-91. 
Federal Task Force on Homelessness and Severe Mental Illness. (1992). Outcasts on main street (ADM 92-1904). Washington, DC: Interagency Council on the Homeless and U. S. Dept. of Health and Human Services.

Goering, P., Wasylenki, D., St. Onge, P., Paduchak, D., \& Lancee, W. J. (1992). Gender differences among clients of a case management program for the homeless. Hospital and Community Psychiatry, 43, 160-165.

Goldman, Howard. (1992, November). Preliminary outcome data from Robert Wood Johnson Demonstration project sites. American Public Health Assocation Conference, Washington, DC.

Hazel, K., Herman, S. E., \& Mowbray, C. T. (1991). Characteristics of adults with serious mental illness in a public mental health system. Hospital and Community Psychiatry, 42, 518-525.

Hensher, C., \& Johnson, L. W. (1981). Applied discrete choice modeling. London: Croom Helm.

Hyde, A. P., \& Goldman, C. (1993). Common family issues that interfere with the treatment and rehabilitation of people with schizophrenia. Psychosocial Rehabilitation Journal, 16, 63-74.

Leda, C., \& Rosenheck, R. (1992). Mental health status and community adjustment after treatment in a residential treatment program for homeless veterans. American Journal of Psychiatry, 149, 1219-1224.

Lefley, H. P. (1993). A family perspective on rehabilitation. In P. W. Flexer \& P. Solomon (Eds.), Psychiatric rehabilitation in practice (pp. 17-30). Boston: Andover.

Lipton, F. R., Nutt, S., \& Sabatini, A. (1988). Housing the homeless mentally ill: A longitudinal study of a treatment approach. Hospital and Community Psychiatry, 39, 40-45.

Marcus, L. R., Cohen, N. L., Nardacci, D., \& Brittain, J. (1990). American Journal of Psychiatry, $147,1557-1561$.

Mercier, C., Fournier, L., \& Peladeau, N. (1992). Program evaluation of services for the homeless: Challenges and strategies. Evaluation and Program Planning, 15, 417-426.

Mowbray, C. T., Bybee, D., \& Cohen, E. (1993). Describing the homeless mentally ill: Cluster analysis results. American Journal of Community Psychology, 21, 67-93.

Mowbray, C. T., Cohen, E., \& Bybee, D. (1991). Services to individuals who are homeless and mentally ill: Implementation evaluation. In D. Rog (Ed.), Evaluating programs for the homeless. New directions for program evaluation (No. 52, pp. 75-90). San Francisco: Jossey-Bass.

Mowbray, C. T., Cohen, E., \& Bybee, D. (1993). The challenge of outcome evaluation in homeless services: Engagement as an intermediate outcome measure. Evaluation and Program Planning, 16, 337-346.

Mowbray, C. T., Cohen, E., Harris, S. N., Trosch, S., Johnson, S., \& Duncan, B. (1992). Serving the homeless mentally ill: Mental Health Linkage. Journal of Community Psychology, 20, 215-227.

Newman, S. J. (1992). The severely mentally ill homeless: Housing needs and housing policy. Baltimore, MD: Johns Hopkins University Institute for Policy Studies (Occasional Paper No. 12).

Rife, J. C., First, R. J., Greenlee, R. W., Miller, L. D., \& Feichter, M. A. (1991). Case management with homeless mentally ill people. Health and Social Work, 16, 58-67.

Rosenheck, R., \& Gallup, P. (1991). Involvement in an outreach and residential treatment program for homeless mentally ill veterans. Journal of Nervous and Mental Disease, 179, 750-754. 
Sosin, M., Piliavin, I., \& Westerfelt, H. (1990). Toward a longitudinal analysis of homelessness. Journal of Social Issues, 46, 157-174.

Steinberg, D., \& Colla, P. (1991). LOGIT: A supplementary module for SYSTAT. Evanston, IL: SYSTAT, Inc.

Toomey, B. G., First, R. J., Rife, J. C., \& Belcher, J. R. (1989). Evaluating community care for homeless mentally ill people. Social Work Research and Abstracts, 25(4), 21-26.

Wright, J. D. (1990). The health of homeless people. Evidence from the National Health Care for the Homeless Program. In P. W. Brickner et al. (Eds.), Under the safety net (pp. 15-31). New York: Norton.

Yeich, S., Mowbray, C. T., Bybee, D., \& Cohen, E. (1994). The case for a supported housing approach: Consumer housing and support preferences. Psychosocial Rehabilitation Journal (in press). 